\title{
Manufacturing, Developments and Constraints in full 3D Printing of Frequency Selective Surface using Low-Cost Open-Source Printer
}

\author{
A. Shastri, B. Sanz-Izquierdo, A. Elibiary and E.A. Parker
}

\begin{abstract}
A comprehensive study of developing a novel printing system using a low-cost open-source printer for fully 3D printing frequency selective surface is presented in this paper. The novel printing setup employs a low-cost printer to print a plastic based filament and a conductive silver ink paste simultaneously. As there were no printers available in the market for this application, the open-source Fused Filament Fabrication (FFF) printer was modified to accommodate two extruders mounted on the same extruder carriage. Techcon TS250 air pressure dispenser was employed for the extrusion of silver ink. Extension pieces for the extruder carriage were also 3D printed using a Fused Deposition Modelling (FDM) printer to reduce the production costs. A bandstop FSS comprising of square loop elements was designed to demonstrate the full fabrication. The FSS operated at a central frequency of $2.55 \mathrm{GHz}$ and provided a good angle of response with wide bandwidths. Surface profiles of the printed FSS and substrate demonstrate the reliable fabrication of the FSS design. This full 3D printing method provides an economical, ecofriendly, swift, reliable, and viable substitute for the fabrication of FSS designs that could be highly customised in terms of fabricating three-dimensional FSS designs with reliable performances. The designs can be printed and deployed to reduce the drop in signal within an enclosed environment.
\end{abstract}

Index Terms - 3D Printing, Additive Manufacturing, Frequency Selective Surfaces, Full 3D Printing, Fused Filament Fabrication, Surface Profiles, Vacuum Extrusion.

\section{INTRODUCTION}

Frequency Selective Surfaces are two-dimensional cyclic array arrangements which deliver a filtering response to the incident electromagnetic signal wave [1], [2]. A planar, twodimensional FSS typically comprises of an array of metallic patches, slots or other recurring structures on a dielectric substrate [2]. Bandstop FSS arrays are regularly deployed within indoor environments and buildings to enhance the signal coverage and strength [3] - [12]. FSS implementations considerably enhance the signal strength by reflecting the undesirable frequencies off their surfaces within the indoor enclosures within the building and letting desired frequencies to go through for fundamental mobile and emergency

This work was funded by UK EPSRC High Value Manufacturing Fellowship (REF: EP/L017121/1), the EPSRC grant titled Low-Profile UltraWideband Wide-Scanning Multi-Function Beam-Steerable Array Antennas (EP/S005625/1), Royal Society - International Exchanges 2019 Cost Share (NSFC) (Ref: IECINSFCl191780) and CHIST ERA WISDOM project under EPSRC grant EP/P015840/1. communications. Magnitude and amounts of a given signal's scattering losses are governed by the dielectric properties, structural design, conductivity, and the types of the recurrent structures of the materials used in the construction of a building. These parameters have all become an integral part of the EM architecture of the building. Bandstop FSS designs have witness tremendous growth over the years and they are now developed using various manufacturing techniques.

$3 \mathrm{D}$ printing or additive manufacturing of EM structures is done by breaking the designs into several layers and depositing the multiple layers of the printing material. Over the past decade, 3D printing of FSS structures [11] - [20] and antennas [21] - [24] are reportedly developed with the help of various 3D printing techniques. Screen printed RF shielding FSS is reported in [11]. Inkjet printing of FSS is reported in [12]. Metamaterial absorber printed with the help of Selective Laser Sintering is reported in [13]. 3D printed bandstop FSS structure is reported in [14] which consisted of disposable unit cell elements printed with the help of Fused Filament Fabrication (FFF). An FSS with dielectric elements printed using Sterolithography (SLA) is reported in [15]. Fused Deposition Modelling (FDM) using reinforced carbon plastic is reported in [16]. Aerosol Jet printing of FSS structures is demonstrated in [17], [18] where bandstop and bandpass structures are printed for RF shielding in windows [17] as well as millimetre wave and low-THz applications [18]. Novel development of the 3D FSS structures are depicted in [19] where 3D printing of a base unit cell structure using plasters was demonstrated with an additional painted layer of conductive material. Further development and expansion were reported in this design by using Fused Deposition modelling (FDM) which reduced the frequency of operation with the help of partially metalized conductive elements of the array [20] that were painted manually.

Some of the EM structures make use of a combination of two or more fabrication techniques. A combination of FDM, partial metalizing and hand painting is reported in [19], [20] whereas a combination of inkjet printing and SLA is reported in [21]. 3D printing of patch antenna using FDM and ultrasonic wire mesh

A. Shastri is currently affiliated with Banasthali Vidyapith, Banasthali, Vidyapith, Rajasthan, India 304022. B. Sanz-Izquierdo, A. Elibiary and E. A. Parker are affiliated with the School of Engineering and Digital Arts, University of Kent, Canterbury CT2 7NT, United Kingdom. (e-mail: anshumanshastri@banasthali.in ; b.sanz@kent.ac.uk; ahmed.ibiary@gmail.com; e.a.parker@kent.ac.uk ) 
embedding technique is depicted in [23]. The printed structures can be further described along the line of printing using expensive equipment [13] - [20], [23] - [24] and low-cost printing equipment [11], [12], [21], and [22].

This paper presents the development of a Low-Cost Openaccess $3 \mathrm{D}$ additive manufacturing system and its application in the production of 3D printed Frequency selective surfaces. The fabrication technique consists of a machine which combines FFF printing of thermoplastic PLA substrate and direct writing of conductive silver ink paste. The printing technique is introduced for the development of EM filtering structures. A microwave bandstop FSS design is printed with the help of the in-house printing equipment. Silver conductive paste was used in tandem with 3D fila-print PLA as the substrate. Magnified profile of the printed square loops and the surface profile demonstrates the effectiveness of the printing setup. The design demonstrated a reasonable angle of incidence response. This FSS design fabrication provides a cost-effective alternative to previously proposed fabrication techniques for full 3D printing FSS using a single machine.

This paper is arranged as follows: Section II describes the fabrication technique and setting up of the printer which was developed for the simultaneous fabrication of the substrate and the FSS. Printer assembly and considerations are also presented in this section. Section III describes the FSS design and simulated transmission response of the design and Section IV presents the fabrication and measured transmission response of the printed FSS. The surface profiles and pictures of the design are also presented within this section. Finally, the concluding remarks are presented in Section V.

\section{Assembly of the 3D PRinting EQUiPMENT}

\section{A. Printer Assembly Considerations}

This FSS design required a printer that could deposit both plastic substrate material and conductive metal simultaneously. As there are no specialized printer available within the existing printer market fulfilling these requirements, the printer had to be built for this application. A number of production and fabrication considerations were taken into account while deciding the printing setup. Firstly, to reduce the cost of the production, a modified open-source printer base frame was preferred instead of a pre-existing multi-step printing bed, which was also not available in the existing market. Secondly, the base frames had their own limitations and constraints in terms of the bed dimensions, printing layer resolution, motor speed and the printing speed. Factors such as printing resolution determines the critical properties such as density and permittivity of the printed plastic base substrate. Thirdly, most of the available open-source low-cost printers supported the fabrication of plastic based PLA and ABS substrates only. Additionally, ABS can only be supported in printers that had a heating bed. Fourthly, to print conductive silver tracks simultaneously using the printer, the metal had to be melted and then deposited in regulated and recurring patterns. The proposed printing setup layout can be seen in Fig. 1.

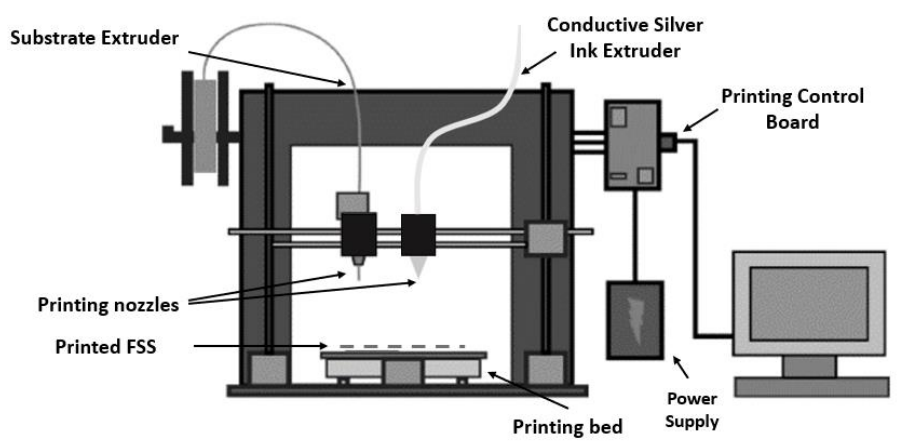

Fig. 1. Proposed 3D printer setup with dual simultaneous printing deposition heads

Since most conductive metals such as silver require extremely high temperatures to be melted, the molten silver ink is then expected to be cooled proximately so that it does not damage the printer particularly as it is required to be deposited in combination with another material such as plastic which has a significantly lower melting temperature. Due to this massive disparity in temperature requirements, this combination of the technique was not viable.

Therefore, to overcome the hurdles, studies into various open-source arrangements were conducted to come up with the Mbot Cube 3D base printer [25] that was a redesigned clone of the Makerbot Replicator [26] which is an open-source printer. To overcome the issues with printing beds and plastic substrates, the designs were made in consideration of the overall build volume size of $200 \times 200 \times 170 \mathrm{~mm}^{3}$ and PLA was selected as the substrate to eradicate the need for a heating bed. The larger printing bed also allowed the fabrication of larger arrays. Finally, to overcome the issues with conductive metallic silver, studies were conducted to provide an unconventional solution using a liquified metallic silver paste and dispensing it through a regulated duct such as a syringe. The silver paste comes from the company Voxel8 [27] which has created a silver nanoparticle ink paste that is augmented for $3 \mathrm{D}$ printing applications. The conductive ink paste possesses an electrical resistivity of under $3 \times 10^{-7} \Omega$-m when cured with a conductance of approximately $2 \times 10^{5} \mathrm{~S} / \mathrm{m}$.

\section{B. Printer Building and Calibration}

As the layout and functioning of the printer had to be altered to accommodate the second extruder, certain components of the printer had to be added to the extruder carriage. The additional plastic extruder motor and an interchangeable syringe extruder were added for simultaneous deposition of PLA and conductive paste. As the extruding unit became larger in size, the end stop dimensions of the motor was reduced accordingly. To reduce the cost of designing, the parts required in the extruder carriage to accommodate the dual operations were $3 \mathrm{D}$ printing using an industrial grade FDM printer. This industrial printer provides a layer resolution of $0.02 \mathrm{~mm}$ to $0.35 \mathrm{~mm}$, which means that it can print some extremely intricate designs with reliable accuracy with high resolutions along with rapid printing speed with a high of $300 \mathrm{~mm} / \mathrm{s}$. 


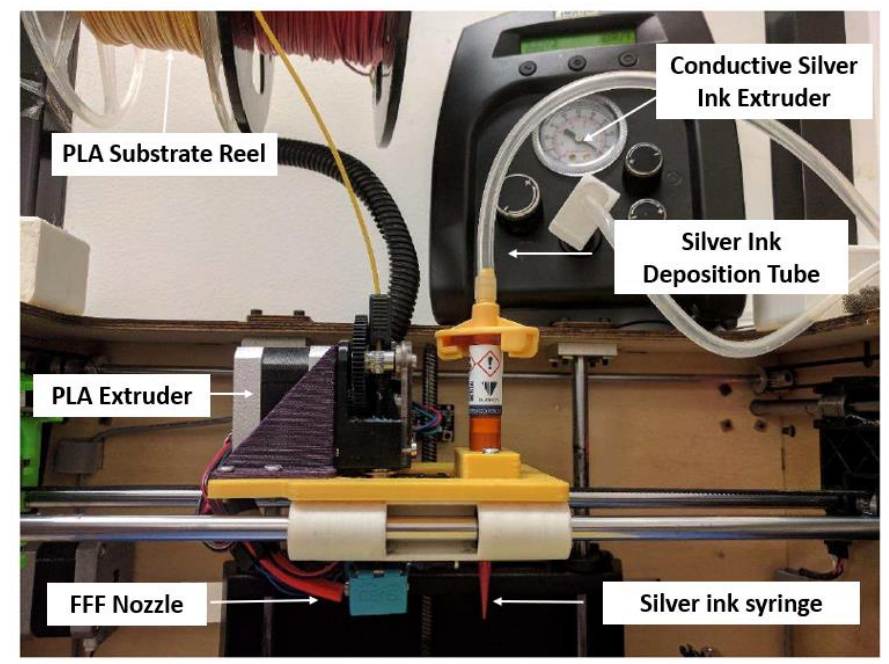

Fig. 2. Assembled 3D printer design setup with dual simultaneous printing deposition heads

The components were designed in CST Microwave Studio ${ }^{\mathrm{TM}}$ and the final designs were exported to an stl file and fed to the slicing software. Some further printing considerations were required for the printing of parts. A $100 \mu \mathrm{m}$ print resolution provides smoothness of the printed surface. A range of 75-80\% of infill helps with the durability of the printed structures, but the durability is significantly high so using $80 \%$ maximum durability is economical as it saves the filament. A perimeter layer limit of two or higher ensures of good binding with far fewer chances of disintegration over time. Using the given considerations, the components were printed and assembled for the printer. The printer with the dual extruders can be seen in Fig. 2. The printing bed was also modified with a heating bed so that filaments like ABS can also be utilised. Two key elements of software that constituted its firmware were designed namely the Marlin firmware and the trigger script to control the second extruder. The Marlin firmware consists of a collection of commands that characterize all the attributes within the printer's system and movement along with its specialised operations for associated devices.

The files were uploaded on the RAMPS 1.4 Printing Control Board to operate the required G-codes. Both the printing extruders had their own individual Arduino ${ }^{\circledR}$ microcontroller devices to control the deposition and their active and inactive states. The silver extruder ink tube as seen on the right side in Fig. 2 consists of a dispersion tube and the voxel8 silver paste casing.

Calibration of the dual extruder printer is an extremely vital aspect of this printer. The initial setup for the printer consisted of assigning the steps per millimetre along all the axis as well as calibrating and realigning the bed height for the modified printer. From the software point of contention, the printing temperature and print speed are required to be in tune according to the individual process, to go with the extrusion multiplier as well as the width of the boundaries. The steps per $\mathrm{mm}$ of extruder motor regulates the feeding quantity of filament to the hot end. $150 \mathrm{~mm}$ of filament is gauged and indicated from the top side of the extruder motor when the extruder is warmed up to the desired temperature. A set of commands is then sent through to the motor to extrude a further $100 \mathrm{~mm}$ length of the filament.

The Techcon TS250 dispenser machine [28] was deployed for the conductive silver ink paste operates through an adjustable output of air pressure that controls and alters the flow of various pastes and conductive inks. An analytical study to determine the optimum pressure flow was conducted. The most optimum track width was obtained when the pressure was between 1-2 bar after varying the pressure from 0.1 bar to 7.9 bar and observe the track widths for all the pressure levels.

With all the calibration and setup work successfully accomplished with reliable and repeatable printing results obtained with the printer, the printer was ready for the printing. A microwave FSS design was developed for printing using the printer setup.

\section{SQUARE LOOP FSS DESIGN}

\section{A. FSS Design}

Passive bandstop FSS arrays function as filtering screens to provide shielding for electronic devices from interferences caused by the ambient signals. Square loop elements are fashionable FSS unit cell elements which exhibit dual polarization along with demonstrating excellent angle of incidence behaviors. The original study on the square loop elements was first reported back in 1982 [29] where the designing and the equivalent circuits of the loops were introduced. The resonant frequency of a square loop is determined by the length of one arm of the loop that is typically $\lambda / 4$ of its wavelength.

The square loop FSS design is presented in Fig. 3 keeping the manufacturing aspects of the printer in mind. The critical dimensions for the design can be seen in Table I. The element was designed on PLA filament-based 3D FilaPrint [30] as substrate with Voxel8 [27] silver ink paste model for the loops and operated at $2.55 \mathrm{GHz}$. The length of the square loop was $26.4 \mathrm{~mm}$ with a track width of $1.25 \mathrm{~mm}$. The thickness of the substrate was $0.8 \mathrm{~mm}$ which was specifically developed in accordance with the printer to make the PLA substrate as thin as possible without damaging the integrity of the print. The gap between the two adjacent elements was $2.55 \mathrm{~mm}$. A number of designs were developed where the distance between the adjacent elements was varied from $2 \mathrm{~mm}$ to $4.5 \mathrm{~mm}$. A parametric study of the designs was conducted, and it was observed that as the gap between the elements was reduced, the capacitance between them increased and the unit cell element operated at a lower frequency. The optimum spacing was found to be $2.55 \mathrm{~mm}$. The simulated transmission response can be seen in Fig. 4. The design operated at a central frequency of $2.55 \mathrm{GHz}$. The $\mathrm{TE}-45^{\circ}$ and $\mathrm{TM}-45^{\circ}$ angle of incidence responses operated at $2.61 \mathrm{GHz}$ and $2.64 \mathrm{GHz}$, respectively.

A shift of approximately $1 \%$ was observed between the transmission responses at the angle of incidence of $0^{\circ}$ and $45^{\circ}$, respectively. The transmission response offered a significantly wide bandwidth of $38 \%$ at the normal response and $37 \%$ for the $\mathrm{TE}-45^{\circ}$ and $\mathrm{TM}-45^{\circ}$ responses. Overall, the transmission response covered a wide range from $2.1 \mathrm{GHz}$ all the way up to almost $3 \mathrm{GHz}$. 


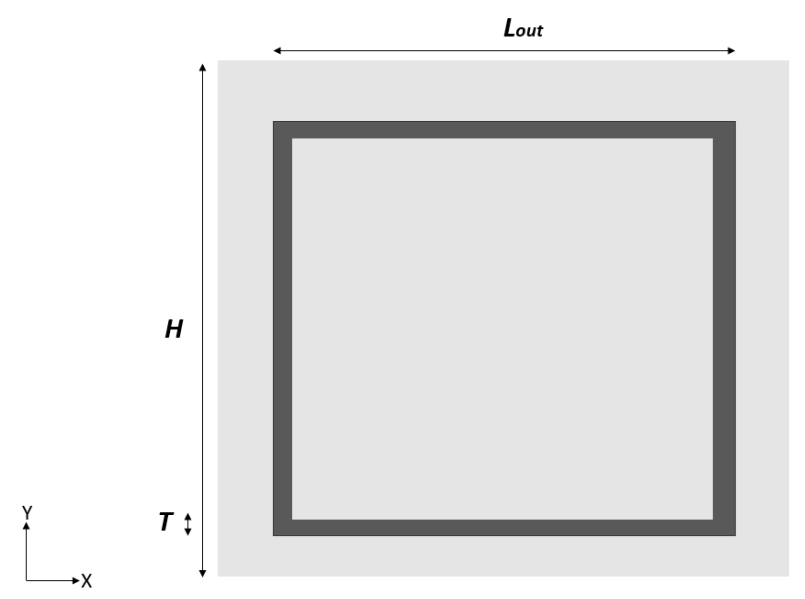

Fig. 3. Square-loop patch FSS unit cell dimensions

TABLE I

FSS UNIT CELL DIMENSIONS

\begin{tabular}{ccccc}
\hline \hline Substrate & Length & $L_{\text {out }}$ & $H$ & $T$ \\
PLA & $\begin{array}{c}\text { Dimensions } \\
(\mathrm{mm})\end{array}$ & 26.4 & 28.95 & 1.25 \\
\hline \hline
\end{tabular}

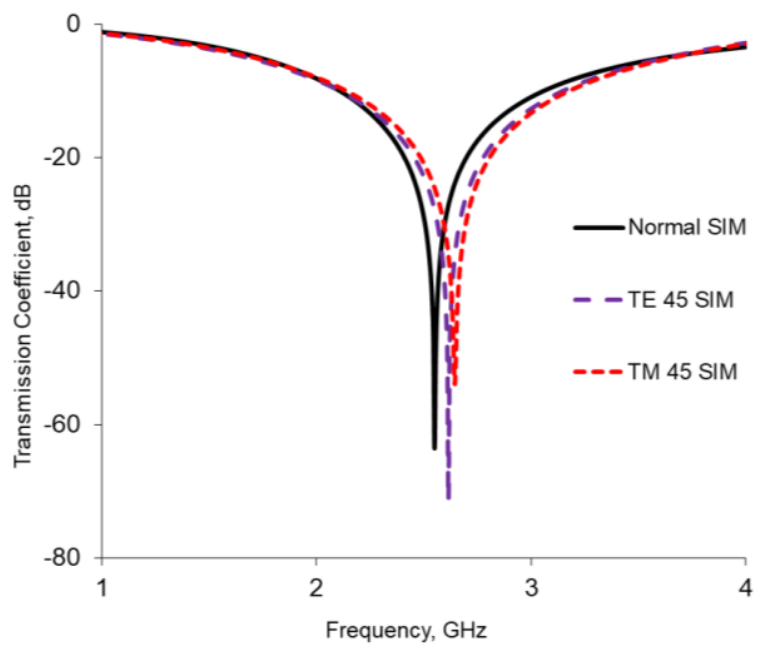

Fig. 4. Simulated Transmission Response

\section{FABRICATION AND MEASUREMENTS}

\section{A. FSS Design Fabrication}

The whole FSS was printed as an array. Keeping the bed size for the printer in mind, an array of $5 \times 6$ was printed as they fitted perfectly. The thickness of the printed PLA substrate was $0.8 \mathrm{~mm}$, and the permittivity was 2.35 . The substrate had $100 \%$ fill in the fabrication for the durability of design. A number of samples were printed. The printing layer height of 200 microns was fed for the design that provided four layers of PLA deposition. The layer height was set at 200 microns for the conductive silver ink. The single layer of deposition was adequate to safeguard the continuity and reliable resistance levels of the printed loops without requiring a further deposition of ink. Regular cleaning safeguarded the nozzle from clogging.

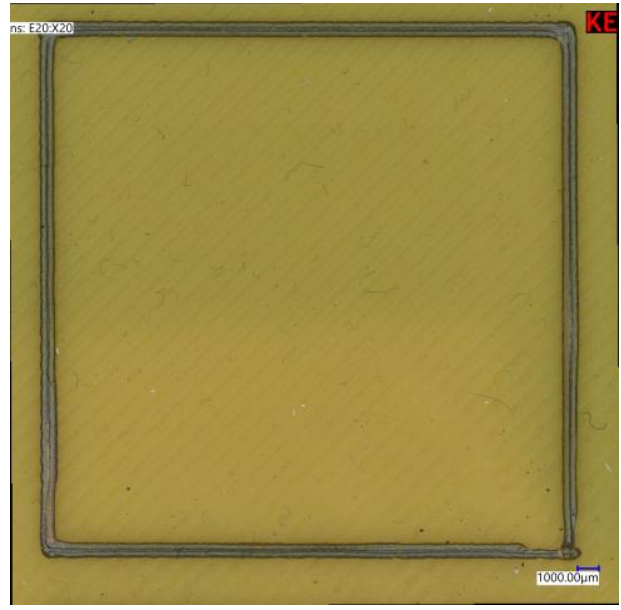

Fig. 5. Printed square loop unit element

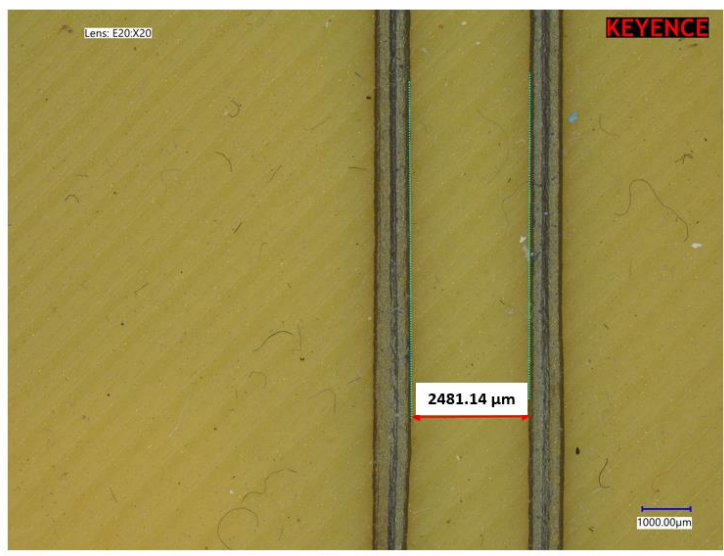

Fig. 6. Gap between two printed loop elements

\section{B. Printed FSS Profile}

A picture of a single loop of the printed FSS design can be seen in Fig. 5. The photographs and profiles were obtained using a Keynce $^{\circledR}$ 4K Ultra HD microscope with 20x magnification and a Talysurf CCI optical interferometer. resolution of the loop element and uniformity can clearly be seen in the photo. Fig. 6 denotes the gap between two elements by further magnifying the profile. Measured gap between two elements was $2481.14 \mu \mathrm{m}$ or $2.48 \mathrm{~mm}$ which was adequately close to the $2.55 \mathrm{~mm}$ gap as per the design. Fig. 7 represents the surface roughness profile map of a magnified segment of the PLA substrate whereas Fig. 8 presents the 3D profile mapping of the printed FSS track. The mean deviation in the printing of the PLA was $7.962 \mu \mathrm{m}$ with a maximum deviation of $12.367 \mu \mathrm{m}$ demonstrating a reliable deposition. The cross-section profile exhibited the printing toolpath which printed the desired loop structure as a combination of inner and outer loop. The proximity of the ink allowed the two loops to fuse thereby forming the single loop with the anticipated dimensions. The low-cost fabrication of the design was completed in about 2.5 hours and did not require drying or curing. The structures could be aligned to create a larger array. The solid, durable PLA base provided longevity to the printed structures. All the printed FSS loops were tested for resistivity and the resistivity for all the designs. As indicated by the manufacturers, Voxel8, remained below the $5 \times 10-7 \Omega-\mathrm{m}$ range [27] with a maximum deviation of $1 \times 10-7 \Omega$-m. 


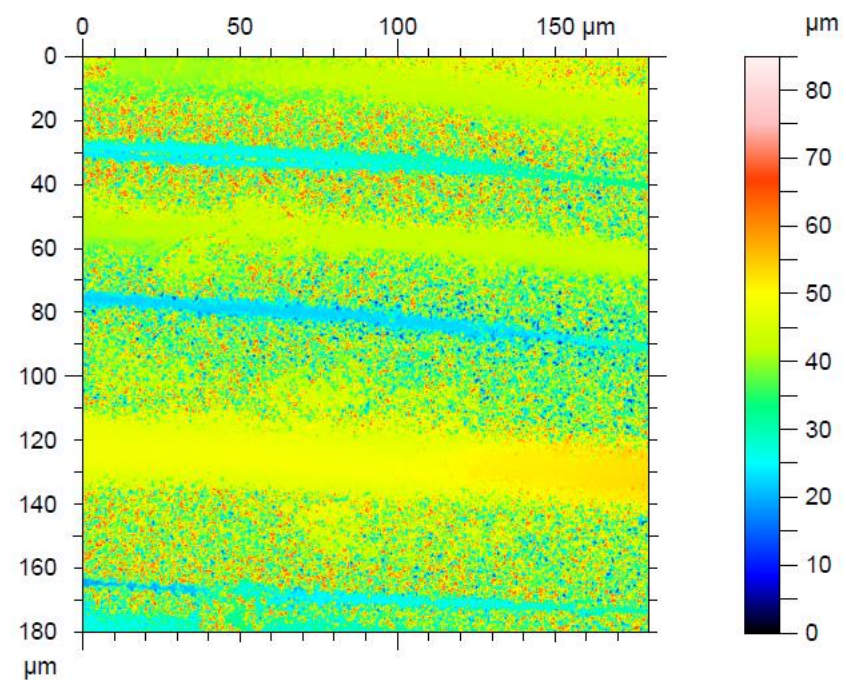

Fig. 7 Surface profile of the printed PLA substrate

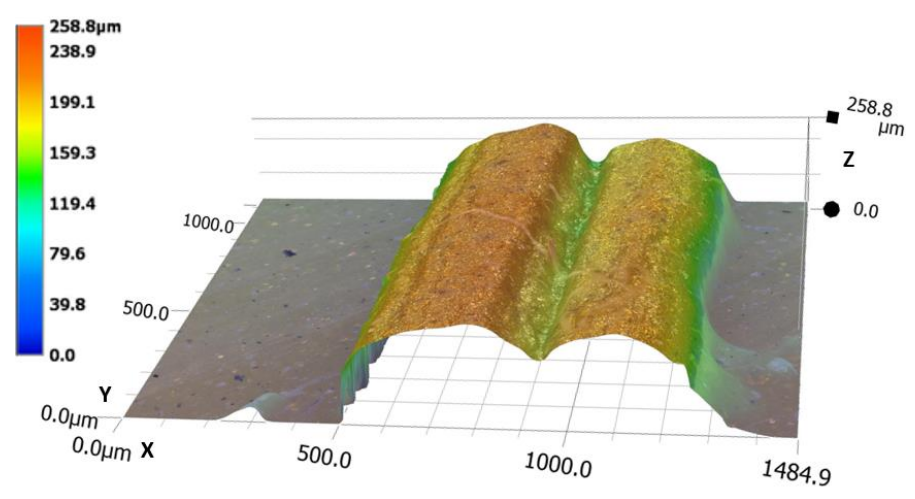

Fig. 8. Measured 3D profile of a printed track and the PLA substrate

\section{Measurement Setup and Results}

The FSS designs were measured individually and by adding a number of printed sub-arrays to constitute a larger array of elements. Mounting two FSS adjacent to each other vertically provided an effective array of $10 \times 6$. The designs were measured in a plain-wave chamber. The measurement setup can be seen in Fig. 9. The FSS structures were mounted in a slot within the absorber screen which could rotate along its axis for angle of measurements. Positioning of the FSS structures within the screen is depicted in Fig. 10. The $10 \times 6$ array created by placing two FSS adjacent to one another that filled up the screen aperture. The measurements were conducted with the help of an Anritsu ${ }^{\circledR}$ 37397C Vector Network Analyzer (VNA).

The FSS was measured for the transmission response. The measured incident transmission responses for the design can be seen in Fig. 11. The design operated at $2.56 \mathrm{GHz}$ which was almost the same as the simulated results. A $28 \%-10 \mathrm{~dB}$ bandwidth was observed for the normal transmission response.

The $\mathrm{TE}-45^{\circ}$ and $\mathrm{TM}-45^{\circ}$ responses operated at $2.59 \mathrm{GHz}$ and $2.65 \mathrm{GHz}$, respectively. The $\mathrm{TM}-45^{\circ}$ response had a shift of $3 \%$ whereas the $\mathrm{TE}-45^{\circ}$ response demonstrated a shift of $2 \%$. All the three responses however presented a significantly wide $-10 \mathrm{~dB}$ bandwidth response with a band over $2.4 \mathrm{GHz}$ covered by all the modes. Overall, the measured responses were in good coherence with the simulated results.

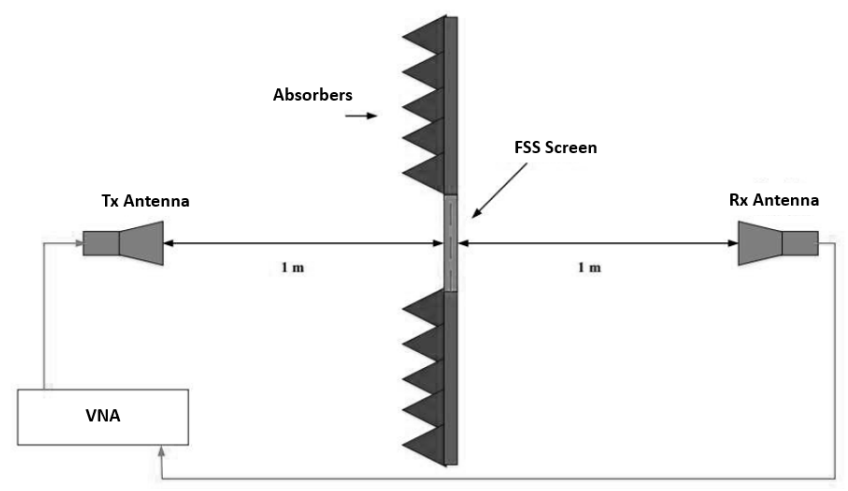

Fig. 9. Measurement Setup Layout

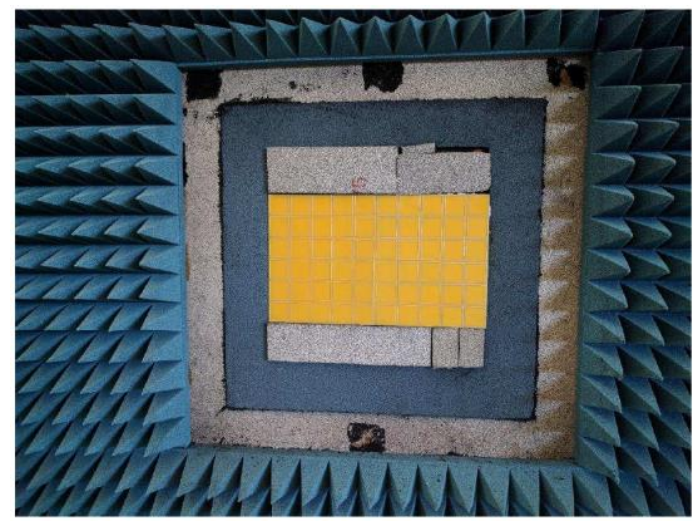

Fig. 10. FSS fitted inside the absorber screen

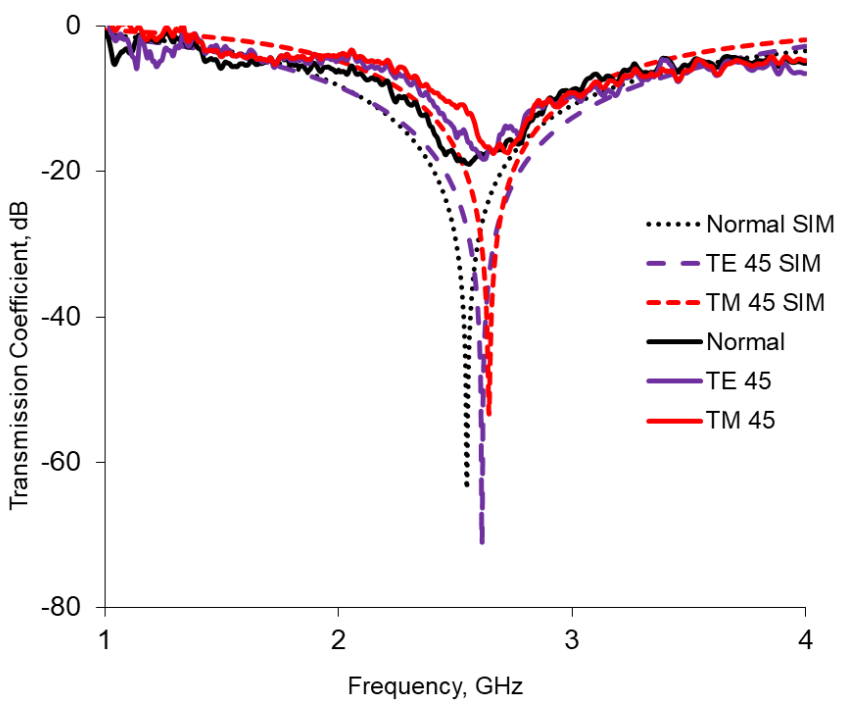

Fig. 11. Measured Transmission Response of the FSS structure

\section{PRINTER LIMITATIONS}

The printed presented a number of limitations along with its developments. The limitations posed challenges and constraints which limited certain aspects of printing certain structures. Firstly, the printer could only print PLA and ABS substrates. Other filaments posed challenges in fabrication. Secondly, the conductive paste was required to be deposited at room temperatures due to the relatively low temperature tolerance of filaments in comparison to conductive metals and inks. Thirdly, the calibration of the system was always prone to human errors. 
TABLE II

COMPARISON BETWEEN THE PERFORMANCE CHARACTERISTICS OF FSS FABRICATION TECHNIQUES

\begin{tabular}{|c|c|c|c|c|c|}
\hline Characteristics & Hand Painting & Screen Printing & Inkjet Printing & Aerosol Jet Printing & Proposed Method \\
\hline References & [19], [20] & [11] & [12] & {$[17],[18]$} & - \\
\hline Substrates & ZP150 & PET & PEL Paper & Glass and Kapton & $\begin{array}{c}\text { PLA } \\
\text { (ABS possible) }\end{array}$ \\
\hline Conductive Materials & Silver ink & Silver ink & $\begin{array}{c}\text { DMC-11610 } \\
\text { Nanoparticle silver } \\
\text { ink }\end{array}$ & $\begin{array}{l}\text { Cabot nanoparticle } \\
\text { silver ink }\end{array}$ & $\begin{array}{l}\text { Voxel8 conductive } \\
\text { silver paste }\end{array}$ \\
\hline Print Resolution & $0.1 \mathrm{~mm}$ & $0.2 \mathrm{~mm}$ & $<0.1 \mathrm{~mm}$ & $5 \mu \mathrm{m}$ & $0.2 \mathrm{~mm}$ \\
\hline Tolerance & $\pm 8 \%$ & $\pm 7 \%$ & $\pm 5 \%$ & $\pm 5 \%$ & $\pm 5 \%$ \\
\hline Permittivity & NA & 3.4 & 4 & 5.27 and 3.4 & 2.35 \\
\hline Variable Permittivity & No & No & No & No & Yes \\
\hline $\begin{array}{c}\text { Resistivity of } \\
\text { conductive tracks }\end{array}$ & $5 \times 10-7 \Omega-\mathrm{m}$ & $2.4 \times 10-7 \Omega-\mathrm{m}$ & $2.4 \times 10-7 \Omega-\mathrm{m}$ & $2.6 \times 10-7 \Omega-\mathrm{m}$ & $4 \times 10-7 \Omega-\mathrm{m}$ \\
\hline Surface roughness & $25 \mu \mathrm{m}$ & $20 \mu \mathrm{m}$ & $10 \mu \mathrm{m}$ & $0.627 \mu \mathrm{m}$ & $12.367 \mu \mathrm{m}$ \\
\hline Rapid prototyping & No & Yes & No & No & No \\
\hline Fabrication steps & Multi & Multi & Single & Multi & Single \\
\hline Scalability & No & Yes & No & No & Yes \\
\hline Cost & $\sim \$ 350$ & $\sim \$ 500$ & $\sim \$ 25,000$ & $\sim \$ 200,000$ & $\sim \$ 250$ \\
\hline Full 3D Printing & No & No & No & No & Yes \\
\hline
\end{tabular}

There were several aspects of analysis and evaluation where reading and observational errors increased the overall tolerance of the system. Fourthly, despite the overall print bed build volume size of $200 \times 200 \times 170 \mathrm{~mm}^{3}$ and the ability to be able to print along the $z$-axis, the maximum printable height was preferred to be approximately $100 \mathrm{~mm}$ while keeping the costefficiency and material wastage in mind. The maximum achievable height was $120 \mathrm{~mm}$. Along with that, multi-layer deposition of the conductive paste was unsuccessful due to the low viscosity of the paste. Fifthly and finally, the printed height of the substrate was optimised to $0.8 \mathrm{~mm}$ to avoid the bending of the PLA substrate after fabrication. Bending was observed due to adhesion of the layer on the print bed and cracks developed during the removal of the printed layer in one unsuccessful prototype. Overall, a number of printer limitations were identified, and they were rectified.

Finally, a comparison table illustrating the fabrication performances and characteristics of various printing techniques in fabricating FSS is shown in table II. The proposed method provided the illusive cost-effective option with full 3D printing of the FSS structures at the lowest cost. The printer could also provide the option of scalability. The option of variable permittivity using PLA and the variation in response can also be beneficial in various applications. 


\section{CONCLUSION}

The development of an additive manufacturing open-source machine and the considerations for fully 3D printed Frequency Selective Surface structure have been discussed. A low-cost printer system combining FFF with an air pressure dispenser was able to print plastic substrates and metallic conductive materials simultaneously. A number of considerations and adjustments were necessary within the printer which included recalibrating the extruder limits and printing components for the printer built using an FDM printer.

A square loop FSS structure was designed and printed while taking the manufacturing constraints of the printing system into account. The printed profile of the FSS demonstrated the success of the fabrication technique. Each fabricated design was tested for conductivity and the designs demonstrated the conductivity in line with the expected values for the ink. The conductivity was achieved by printing just a single layer of the conductive paste and can be increased further by adding additional layers through various processes. The FSS operated at a central frequency of $2.55 \mathrm{GHz}$ and provided a significantly stable angle of incidence response with all the modes of the angle of response covering the $2.4 \mathrm{GHz}$ band while providing wide $-10 \mathrm{~dB}$ bandwidths.

The scalable property of the printing setup makes it a lucrative option for the development of 3D structures which are printed in their entirety. The reliable performance of the ink could provide a potential solution of the development of fully 3D printed FSS designs, and antennas fabricated on a variety of three-dimensional substrates while significantly reducing the cost of fabrication. The 3D printed designs can also be made flexible. With an ongoing research on using 3D conductive filaments, a range of other possibilities using the system are also being explored.

\section{ACKNOWLEDGMENT}

The authors would like to thank Simon Jakes, Antonio Mendoza and Dr. Mike Green of University of Kent for their support during fabrication and measurements. This work was funded by UK EPSRC HVMC Fellowship (REF: $\mathrm{EP} / \mathrm{L} 017121 / 1$ ), the EPSRC grant "Low-Profile UltraWideband Wide-Scanning Multi-Function Beam-Steerable Array Antennas" (EP/S005625/1), Royal Society International Exchanges 2019 Cost Share (NSFC) (Ref: IECINSFC 191780) and CHIST ERA WISDOM project under EPSRC grant EP/P015840/.

\section{REFERENCES}

[1] B. A. Munk, Frequency selective surfaces: Theory and design, New York: Wiley, 2000, pp 636-659, doi: 10.1002/0471723770.

[2] E. A. Parker, "The Gentleman's Guide to Frequency Selective Surfaces," 01-Jan-1991. [Online]. Available: https://kar.kent.ac.uk/59863/. [Accessed: 01-Jun-2020].

[3] F. Costa and A. Monorchio, "A Frequency Selective Radome With Wideband Absorbing Properties," in IEEE Transactions on Antennas and Propagation, vol. 60, no. 6, pp. 2740-2747, June 2012, doi: 10.1109/TAP.2012.2194640.

[4] S. Chakravarty, R. Mittra and N. R. Williams, "Application of a Microgenetic Algorithm (MGA) to the Design of Broad-band Microwave Absorbers using Multiple Frequency Selective Surface
Screens Buried in Dieletrics", IEEE Transactions on Antennas and Propagation, vol. 50, pp. 284-296, 2002.

[5] G.I. Kiani, A.R. Weily and K.P. Esselle, "A novel absorb/transmit FSS for secure indoor wireless networks with reduced multipath fading," IEEE Microw. Wireless Compon. Lett., vol. 16, no. 6, pp. 378-380, 2006.

[6] S. N. Azemi and W. S. T. Rowe, "Development and analysis of 3D Frequency Selective Surfaces," Asia-Pacific Microwave Conference 2011, 2011, pp. 693-696.

[7] M. Raspopoulos and S. Stavrou, "Frequency Selective Buildings Through Frequency Selective Surfaces," in IEEE Transactions on Antennas and Propagation, vol. 59, no. 8, pp. 2998-3005, Aug. 2011, doi: 10.1109/TAP.2011.2158779.

[8] C. Mias, C. Tsokonas, and C. Oswald, An investigation into the feasibility of designing frequency selective windows employing periodic structures 2002, Nottingham Trent University, Nottingham, U.K., Tech. Rep. AY3922.

[9] M. Gustafsson, A. Karlsson, A. Rebelo, and B. Widenberg, "Design of Frequency Selective Windows for Improved Indoor-Outdoor Communication," IEEE Transactions on Antennas and Propagation, vol. 54, no. 6, pp. 1897-1900, 2006.

[10] P. Taylor, E. Parker, and J. Batchelor, "Experimental phase plate employing a phase modulated active frequency selective surface", Microwave and Optical Technology Letters, vol. 52, no. 10, pp. 2300-2302, 2010. Available: 10.1002/mop.25484.

[11] A. A. Dewani, S. G. Okeefe, D. V. Thiel, and A. Galehdar, "Window RF Shielding Film Using Printed FSS," IEEE Transactions on Antennas and Propagation, vol. 66, no. 2, pp. 790-796, 2018.

[12] B. M. Turki et al., "Significant Factors in the Inkjet Manufacture of Frequency-Selective Surfaces," in IEEE Transactions on Components, Packaging and Manufacturing Technology, vol. 6, no. 6, pp. 933-940, June 2016, doi: 10.1109/TCPMT.2016.2561972.

[13] D. Zhou, X. Huang and Z. Du, "Analysis and Design of Multilayered Broadband Radar Absorbing Metamaterial Using the 3-D Printing Technology-Based Method," in IEEE Antennas and Wireless Propagation Letters, vol. 16, pp. 133-136, 2017, doi: 10.1109/LAWP.2016.2560904.

[14] D. Lim, S. Yu and S. Lim, "Miniaturized Metamaterial Absorber Using Three-Dimensional Printed Stair-Like Jerusalem Cross," in IEEE Access, vol. 6, pp. 43654-43659, 2018, doi: 10.1109/ACCESS.2018.2862160.

[15] S. Cho, S. Yoon and I. Hong, "Design of Three-Dimensional Frequency Selective Structure With Replaceable Unit Structures Using a 3-D Printing Technique," in IEEE Antennas and Wireless Propagation Letters, vol. 17, no. 11, pp. 2041-2045, Nov. 2018, doi: 10.1109/LAWP.2018.2871175.

[16] R. Mirzavand, M. M. Honari, S. Aslanzadeh, H. Saghlatoon and P. Mousavi, "Evaluation of One-Staged 3-D Printed Frequency Selective Surface Using Carbon-Fiber-Reinforced Thermoplastic Composite," in IEEE Transactions on Components, Packaging and Manufacturing Technology, vol. 9, no. 11, pp. 2298-2304, Nov. 2019, doi: 10.1109/TCPMT.2019.2917197.

[17] A. Shastri et al., "Evaluation of Aerosol Jet Printing of Frequency Selective Surface on Glass for Building and RF Applications," 2020 14th European Conference on Antennas and Propagation (EuCAP), Copenhagen, Denmark, 2020, pp. 1-5, doi: 10.23919/EuCAP48036.2020.9135346.

[18] A. Shastri et al., "3D Printing of Millimetre Wave and LowTerahertz Frequency Selective Surfaces Using Aerosol Jet Technology," in IEEE Access, vol. 8, pp. 177341-177350, 2020, doi: 10.1109/ACCESS.2020.3024584.

[19] B. Sanz-Izquierdo and E. A. Parker, "3-D Printing of Elements in Frequency Selective Arrays," in IEEE Transactions on Antennas and Propagation, vol. 62, no. 12, pp. 6060-6066, Dec. 2014, doi: 10.1109/TAP.2014.2359470.

[20] B. Sanz-Izquierdo and E. A. Parker, "Frequency selective surfaces formed by partially metalising 3D printed shapes," 2015 9th European Conference on Antennas and Propagation (EuCAP), Lisbon, 2015, pp. 1-4.

[21] J. Heirons, S. Jun, A. Shastri, B. Sanz-Izquierdo, D. Bird, L. Winchester, L. Evans and A. McClelland, "Inkjet Printed GPS antenna on a 3D printed substrate using low-cost machines," 2016 Loughborough Antennas \& Propagation Conference (LAPC), Loughborough, 2016, pp. 1-4, doi: 10.1109/LAPC.2016.7807590. 
[22] A. Shastri, S. Jun, B. Sanz-Izquierdo, H. Aldawas, Q. Ahmed and M. Sobhy, "Evaluation of a low-cost inkjet-printed slot antenna for energy harvesting applications," 2016 Loughborough Antennas \& Propagation Conference (LAPC), Loughborough, 2016, pp. 1-5, doi: 10.1109/LAPC.2016.7807593.

[23] M. Liang, C. Shemelya, E. MacDonald, R. Wicker, and H. Xin, “3D printed microwave patch antenna via fused deposition method and ultrasonic wire mesh embedding technique," IEEE Antennas Wireless Propag. Lett., vol. 14, pp. 1346-1349, 2015.

[24] R. Xu et al., "140 GHz Additive Manufacturing Low-Cost and High-Gain Fabry-Perot Resonator Antenna," 2020 International Workshop on Antenna Technology (iWAT), Bucharest, Romania, 2020, pp. 1-4, doi: 10.1109/iWAT48004.2020.1570598322.

[25] [online] http://www.mbot3d.com [Accessed 24 Aug. 2020].

[26] [online] https://www.makerbot.com [Accessed 25 Aug. 2020].

[27] [online] www.voxel8.co [Accessed 25 Aug. 2020].

[28] "Digital Fluid Dispenser - TS250", Techconsystems.com, 2020. [Online]. Available: http://www.techconsystems.com/en/fluiddispensers/digital-fluid-dispensers/basic-digital-dispensers/ts250digital-fluid-dispenser/ . [Accessed: 25- Aug- 2020].

[29] R. J. Langley and E. A. Parker, "Equivalent circuit model for arrays of square loops," in Electronics Letters, vol. 18, no. 7, pp. 294-296, 1 April 1982.

[30] PLA", Shop.3dfilaprint.com, 2020. [Online]. Available: https://shop.3dfilaprint.com/pla-199-c.asp . [Accessed: 25- Aug2020].

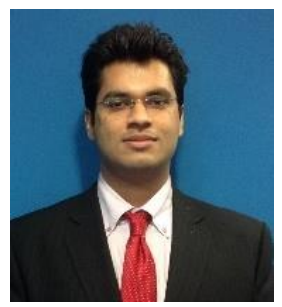

Anshuman Shastri completed his B.E. (Hons.) degree in Electronics and Communications Engineering from Birla Institute of Technology and Science, India in 2014, the MSc degree in Electronics Engineering from University of Kent, U.K. in 2016 and his $\mathrm{PhD}$ in Electronics Engineering from University of Kent, U.K. in 2020.

$\mathrm{He}$ is currently an Associate Professor at School of Automation, Banasthali Vidyapith, India. His research interests include microwave antennas, smart energy harvesting, additive manufacturing, frequency selective surfaces, millimetre wave devices and modelling of frequency reconfigurable structures. He received the International Young Scientist Award in 2020 along with International Young Researcher and Research Excellence Awards in 2021.

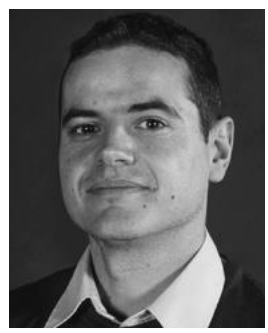

Benito Sanz Izquierdo received the B.Sc. from ULPGC (Spain), and the M.Sc. and Ph.D. degrees from the University of Kent, U.K., in 2002 and 2007 respectively.

From 2003 to 2012, he was Research Associate with the School of Engineering, University of Kent, he became a lecturer in Electronic Systems in 2013 and Senior Lecturer in 2019. In 2012, he worked for Harada Industries Ltd where he developed novel antennas for the automotive industry. His research interests are multiband antennas, wearable microwave devices, additive manufacturing, RF sensing, electromagnetic band-gap structures and frequency selective surfaces.

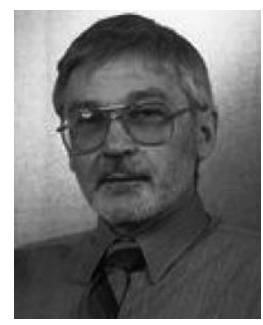

Edward (Ted) A. Parker received the M.A. degree in physics and the Ph.D. degree in radio astronomy from St. Catharine's College, Cambridge University, Cambridge, U.K. He was appointed as a Reader with the University of Kent, Canterbury, U.K., in 1977, where he has been a professor of radio communications, since 1987. He is currently a Professor Emeritus with the University of Kent. He established the Antennas Group, Electronics Laboratory, University of Kent. He is a member of the Livery of the Worshipful Company of Scientific Instrument Makers, London, U.K. His current research interests include the application of frequency selective surfaces and the study and overhaul of antique clocks. Dr. Parker is a member of the Institution of Engineering and Technology. 